\title{
Valoración de puestos de trabajo con perspectiva de género
}

\author{
Gender-neutral job evaluation
}

\author{
M. BEGoÑa GaRcía GIL * \\ Profesora de Derecho del Trabajo y la Seguridad Social \\ Universidad Rey Juan Carlos. Madrid \\ ORCID ID: 0000-0003-1282-9687
}

Recibido: $19 / 03 / 2021$

Aceptado: 26/03/2021

doi: https://doi.org/10.20318/femeris.2021.6136

\begin{abstract}
Resumen. La desigualdad entre hombres y mujeres en las relaciones laborales y su repercusión materializada en la brecha salarial exige una perspectiva de análisis específica vinculada a la valoración de puestos de trabajo. En este estudio realizamos un estudio del procedimiento que sirve de herramienta garantizadora de la igualdad entre mujeres y hombres relativa a la valoración de puestos de trabajo. En concreto, nos referimos a todos aquellos aspectos que relacionados con factores y subfactores de valoración permiten identificar, corregir y evitar sesgos de género que son causa de discriminación indirecta y que provocan desigualdades retributivas.

Palabras clave: valoración puestos de trabajo; igualdad; género.

Abstract. Nequality between men and women in labour relations and its impact on job evaluation Specifically, we refer to the aspects related to assessment factors that allow the identification, correction, and avoidance of gender biases. The wage gap produced due to indirect discrimination requires a specific analysis linked to job evaluation. This study addresses the procedure as a tool to ensure gender equality.

Keywords: job evaluation; equality; gender.
\end{abstract}

\section{Valoración de puestos de trabajo y su relación con la igualdad entre mujeres y hombres}

La valoración de puestos de trabajo es un sistema de tasación y graduación de puestos de trabajo dentro de una organización empresarial mediante el que se asigna a cada puesto un conjunto de elementos específicos: tareas, esfuerzos o requerimientos necesarios para su desempeño.

*begona.garcia@urjc.es 
En relación con la igualdad entre mujeres y hombres la valoración de puestos de trabajo es una herramienta eficaz y eficiente puesto que mide el desempeño del puesto de trabajo desde la neutralidad y la objetividad. De la misma forma sirve para identificar las situaciones de desigualdad entre mujeres y hombres en el ámbito laboral que impacta especialmente en la retribución.

Es obvio, que mujeres y hombres no perciben la misma remuneración por la realización del mismo trabajo o por trabajos distintos, pero de igual valor. Así queda constatado en las cifras y datos que reflejan que la brecha salarial por razón de género es, hoy por hoy, una realidad que aún exige corrección y medidas.

Por situarnos en el estado de la realidad en nuestro país, es importante hacer referencia a las estadísticas que sobre la materia reflejan en los datos la situación general, entre ellos, los que facilita el Instituto Nacional de Estadística (en adelante, INE) en la última encuesta de Estructura Salarial del año 2017 donde se significa que la brecha salarial entre hombres y mujeres se sitúa en torno a un $21,9 \%$.

La Confederación Española de Organizaciones Empresariales (en adelante, CEOE) en el Informe denominado Análisis de la Brecha Salarial de género en España, estableció que las mujeres cobran de media un 12,2\% menos que los hombres, si bien se destacó que entre 2002 y 2014 esa brecha se redujo un 28,06\%.

La Organización Mundial del Trabajo (en adelante, OIT) publicaba el 2 de diciembre del 2020 su Informe Mundial sobre Salarios 2020-2021: Los salarios y el salario mínimo en tiempos de la COVID-19, en este informe se destaca que no hay datos publicados ni suficientes para calibrar el impacto exacto sobre los salarios en el 2020 pero advierte un hecho evidente: "las mujeres se han visto afectadas de manera desproporcionada de muchas maneras, lo que podría ampliar las brechas de género en el mercado laboral y posiblemente acabar con los avances logrados en los últimos años".

Los sindicatos apoyan esta afirmación, al punto que la Central Sindical Independiente y de Funcionarios (en adelante, CSIF) considera que la brecha en el 2020 podría haber subido del 22\% que registró la Agencia Tributaria en el 2019 al 23\% debido a la mayor precariedad laboral.

El INE en la última Encuesta Anual de Estructura Salarial determina que la brecha salarial por ocupaciones en las profesiones aumentó a partir de la Gran Recesión del 2008 pues escaló del 15,17\% en ese año a bordear el 18-19\% en el periodo 2010-2014 y volver a aplanarse desde entonces hasta situarse en el 15,01\% más reciente el año pasado.

Si bien la brecha salarial en profesiones se redujo en el espacio temporal anterior a la pandemia, tenemos que afirmar que tras la misma se ha pronunciado. Al punto que en las actividades profesionales, científicas y técnicas fue del 30,70\%, medio punto porcentual menos que el dato previo, y en las actividades sanitarias y sociales fue el 28,02\% más de un punto porcentual inferior que el año anterior. Un dato significativo e indiscutible es que la brecha es mayor en puestos de mayor responsabilidad.

Tanto en el ámbito comunitario como nacional se han determinado normativamente aspectos significativos que de modo breve podemos señalar. 
En este sentido la Comisión Europea, en su Recomendación de 7 de marzo de $2014^{1}$ sobre el refuerzo del principio de igualdad de retribución entre hombres y mujeres a través de la transparencia instó a los Estados miembros de la UE a promover el desarrollo y uso de sistemas de evaluación y clasificación de empleos no sexistas en su calidad de empleadores, y además propuso como instrumento eficaz una movilización que anime a las empresas e interlocutores sociales a introducir dichos sistemas de evaluación y clasificación de empleos no sexistas. La Comisión Europea recomendó basarse ${ }^{2}$ en el documento de trabajo de los servicios de la Comisión, que acompañaba al informe sobre la aplicación de la Directiva 2006/54/CE ${ }^{3}$ consideraba los métodos de evaluación analíticos (cuantitativos) como los más apropiados para realizar una valoración de puestos desde el punto de vista de la igualdad de género, permitiendo establecer uno de los componentes más importantes del principio de igualdad salarial ${ }^{4}$ denominado "trabajo de igual valor".

En el plano interno, en esta misma línea el Instituto de la Mujer y para la Igualdad de Oportunidades, en el marco del Plan Estratégico de Igualdad de Oportunidades 20142016, y en concreto, del Plan Especial para la Igualdad de Mujeres y Hombres en el ámbito laboral y contra la Discriminación Salarial 2014-2016, puso a disposición de las empresas, en febrero de 2015, una Herramienta de autodiagnóstico de brecha salarial de género ${ }^{5}$. el ordenamiento laboral se refiere al valor del trabajo en los arts. 22 apartados 2 y 3 y en el art. 28 ET con dos efectos.

Por un lado, la determinación de los factores determinantes del grupo profesional que lógicamente marcan la valoración del puesto de trabajo: la agrupación de puestos en función de aptitudes profesionales, titulaciones y contenido general de la prestación, y podrá incluir distintas tareas, funciones, especialidades profesionales o responsabilidades asignadas al trabajador.

Por otro lado, la garantía de la igualdad retributiva entre sexos, definiendo los grupos profesionales ajustados a criterios y sistemas que tengan como objeto garantizar la ausencia de discriminación, tanto directa como indirecta, entre mujeres y hombres en la misma línea y referida a los mismos factores: naturaleza de las funciones o tareas efectivamente encomendadas, así como a las condiciones educativas, profesionales o de formación exigidas para los puestos de trabajo en relación con el desempeño y las condiciones laborales.

La situación actual puede considerarse en progreso, parece que se ha dado un paso importante en los últimos tiempos sobre esta materia, aunque todavía tiene que materializarse.

\footnotetext{
${ }^{1}$ (2014/124/UE) Disponible en: http://www.boe.es/doue/2014/069/L00112-00116.pdf

${ }^{2}$ http://ec.europa.eu/justice/gender-equality/files/gender_pay_gap/swd-2013-_512-final_en.pdf

${ }^{3}$ Directiva 2006/54/CE del Parlamento Europeo y del Consejo, de 5 de julio de 2006, relativa a la aplicación del principio de igualdad de oportunidades e igualdad de trato entre hombres y mujeres en asuntos de empleo y ocupación http://eur-lex.europa.eu/LexUriServ/LexUriServ.do?uri=0J:L:2006:204:0023:0036:es:PDF.

${ }^{4}$ Pons Parera E.: El reconocimiento del derecho a la igualdad retributiva por los ordenamientos internacional, europeo y estatal, en Un futuro sin brecha, Bomarzo 2015. págs.. 24 y ss. y Marie-Thérèse Chicha; Oficina Internacional del Trabajo - Ginebra: OIT, 2008, Disponible: http://www.ilo.org/public/libdoc/ilo/2008/108B09_271_span.pdf

${ }^{5}$ Directiva 2006/54/CE del Parlamento Europeo y del Consejo, de 5 de julio de 2006, relativa a la aplicación del principio de igualdad de oportunidades e igualdad de trato entre hombres y mujeres en asuntos de empleo y ocupación http://eur-lex.europa.eu/LexUriServ/LexUriServ.do?uri=0J:L:2006:204:0023:0036:es:PDF
} 
El desarrollo reglamentario del Real Decreto-ley 6/2019, de 1 de marzo, de medidas urgentes para garantía de la igualdad de trato y de oportunidades entre mujeres y hombres en el empleo y la ocupación (en adelante RDL 6/2019) introdujo, una nueva redacción del art. 28 ET, limitando los factores operantes en materia de valoración de puestos de trabajo a cuatro: condiciones educativas y profesionales, desempeño y condiciones laborales. Con el propósito necesario de establecer los límites y diseño del trabajo de igual valor. Es decir, trabajo en el que coinciden las funciones o tareas o contenido de la activad desempeñada por el trabajador que tiene que establecerse en la ley o en el convenio colectivo. En puestos esencialmente iguales con funciones o tareas idénticas la discriminación por género no es posible.

De la misma forma y desde una perspectiva aún mas sencilla, las condiciones educativas establecen niveles predelimitados normativamente ${ }^{6}$ relacionados conforme al art. 28 ET que debe guardar relación con el desarrollo de la actividad siempre que el empresario precise que el trabajador/a tenga un nivel educativo para el desempeño de sus funciones laborales. En este aspecto debe aplicarse especial cuidado ya que las titulaciones universitarias o de formación profesional pueden establecer diferencias salariales vinculadas con actividades tradicionalmente feminizadas o masculinizadas.

Las condiciones profesionales y de formación, según el RDL 6/2019, sirven para acreditar la cualificación de la persona trabajadora, incluyendo la experiencia o la formación no reglada, siempre que tenga conexión con el desarrollo de la actividad. Esto engloba tanto la experiencia o conocimientos adquiridos por la práctica a lo largo de la carrera de la profesional del trabajador/a y el aprendizaje al margen del sistema reglado.

Para la valoración de aspectos como la responsabilidad o las condiciones laborales es necesario introducir un proceso neutro de valoración de puestos de trabajo, de lo contrario como la norma no establece un sistema específico puede suponer discriminación de género.

Adelantándonos a nuestro análisis, por ejemplo, podemos observar que el TJUE, respecto de la experiencia, ha dejado claro que en supuesto de utilizar el criterio de la antigüedad como factor que contribuye a la fijación de las retribuciones redunde en disparidades de retribución, para un mismo trabajo o para un trabajo de igual valor, entre los trabajadores y las trabajadoras debe interpretarse en el sentido de que es un factor o criterio idóneo para alcanzar la finalidad legítima de recompensar la experiencia adquirida que coloca al trabajador en condiciones de cumplir mejor sus tareas. Téngase en cuenta que el empresario no está obligado a justificar de un modo especial que la utilización de dicho criterio sea idónea para alcanzar la citada finalidad en lo que atañe a un puesto de trabajo determinado, a no ser que el trabajador facilite datos que puedan hacer nacer dudas fundadas a este respecto. Cuando para la fijación de la retribución se utilice un sistema de clasificación profesional fundado en una evaluación del trabajo que debe realizarse, no es necesario acreditar que un trabajador considerado individualmente haya adquirido,

\footnotetext{
${ }^{6}$ Clasificación Nacional de Educación (CNED-2014), basada a su vez en la Clasificación Internacional Normalizada de Educación de la UNESCO (CINE-2011 o ISCED-2011, en su denominación anglosajona), clasificación oficial (la CNED-F).
} 
durante el período pertinente, una experiencia que le haya permitido desempeñar mejor su trabajo ${ }^{7}$.

En cuanto a las condiciones laborales directamente relacionadas con el desempeño, la norma tampoco establece definición ni factores relevantes que indiquen cómo valorar el desempeño del puesto de trabajo.

Por ello de nuevo, el uso del sistema de valoración de puestos resulta una herramienta imprescindible para delimitar qué factores son relevantes para el desempeño de cada actividad. Partiendo de los criterios de "adecuación, totalidad y objetividad", ni es preciso que se tomen necesariamente en consideración los indicados en la norma, ni se impide que se preste atención a otros que puedan resultar adecuados.

El RDL 6/20198 introdujo que para el uso de la valoración de los puestos de trabajo se tengan en cuenta obligatoriamente tres criterios imprescindibles para determinar que estamos ante trabajos de igual valor independientemente del género de la persona trabajadora: "Un trabajo tendrá igual valor que otro cuando la naturaleza de las funciones o tareas efectivamente encomendadas, las condiciones educativas, profesionales o de formación exigidas para su ejercicio, los factores estrictamente relacionados con su desempeño y las condiciones laborales en las que dichas actividades se llevan a cabo en realidad sean equivalentes".

Dicho de otra forma, el estatuto fija el margen de análisis de los puestos de trabajo centrando la valoración en los denominados factores relevantes relacionados con la actividad. Entre los que se encuentra la equivalencia de condiciones o circunstancias entre trabajos de igual valor tomando en consideración las circunstancias de las personas trabajadoras de uno y otro sexo, de tal forma que cuando concurran de forma desequilibrada en favor de un sexo no deben tenerse en cuenta puesto que el efecto, en caso contrario, provocaría desigualdades salariales.

En la misma medida parece que el registro salarial ${ }^{9}$ y la auditoría salarial ${ }^{10}$, son dos instrumentos que nos permitirán acercarnos a la realidad de cada empresa. Ambos aprobados por Real Decreto en marzo de 2019 y desarrollados en el Real Decreto 902/2020, de 13 de octubre, de igualdad retributiva entre mujeres y hombres ${ }^{11}$ y que entrará en vigor el próximo 14 de abril de 2021. Reglamento de Igualdad Retributiva, aprobado el 13 octubre de 2020 en Consejo de Ministros, fruto del Diálogo Social de gobierno, organizaciones sindicales y empresariales. Este Reglamento, contiene el desarrollo de cómo llevar a cabo las dos herramientas. Establecimiento de un plan de actuación para la corrección de las desigualdades retributivas: con determinación de objetivos, actuaciones concretas, cronograma y persona o personas responsables de su implantación y seguimiento. El plan

\footnotetext{
${ }^{7}$ STJUE $2006 \backslash 2803$ octubre 2006.

${ }^{8}$ DE JESÚS SILVANO, S.M.: Los planes de igualdad en las empresas tras su reciente desarrollo reglamentario, Diario La Ley, núm. 9746, 2020.

${ }_{9}^{9}$ MARTÍNEZ BOTELLO, P.: EL Registro Retributivo: Una herramienta para paliar la brecha salarial entre mujeres y hombres, Revista Aranzadi Doctrinal, ISSN 1889-4380, núm. 3, 2021.

${ }^{10}$ FERNÁNDEZ NIETO, L.A.: El registro obligatorio de salarios y las auditorías salariales como medidas disuasorias de la discriminación retributiva, Diario la Ley, núm. 9765, 2021.

${ }^{11}$ ALONSO ARANDA, M.: La igualdad retributiva entre mujeres y hombres (Análisis normativo del RD 902/2020, de 13 octubre), BIB 2020 \36626c y Callau Dalmau, P.; La aplicación del principio de igualdad de retribución contemplado en el Real Decreto 902/2020, de 13 de octubre. Referentes supranacionales y alcance normativo, Revista del Derecho del Trabajo y la Seguridad Social, núm. 58, 2021.
} 
de actuación deberá contener un sistema de seguimiento y de implementación de mejoras a partir de los resultados obtenidos.

De manera paralela debe referirse esta cuestión en nuestro ordenamiento a la circunstancia de que el 14 de enero de este año ha entrado en vigor el Reglamento de Planes de Igualdad, aprobado también el 13 de octubre pasado en Consejo de ministros y también fruto del Diálogo Social tripartito. A partir de marzo de 2021 las empresas con más de 100 trabajadores y trabajadoras estarán obligadas a negociar un Plan de Igualdad, como se estableció en el Real Decreto de marzo de 2019, y posteriormente en el Real Decreto $901 / 2020$, de 13 de octubre, por el que se regulan los planes de igualdad ${ }^{12}$ y su registro y se modifica el Real Decreto 713/2010.

El Real Decreto 902/2020, de 13 de octubre, de igualdad retributiva entre mujeres y hombres con entrada en vigor 14 de abril de 2021, desarrolla reglamentariamente el artículo 28 del ET. Las principales novedades relativas a la correcta valoración de los puestos de trabajo se establecen en el art. 9 del texto que de acuerdo con lo previsto en el artículo 22.3 del ET, con el objetivo de comprobar que la definición de los grupos profesionales se ajusta a criterios y sistemas que garantizan la ausencia de discriminación directa e indirecta entre mujeres y hombres y la correcta aplicación del principio de igualdad de retribución por trabajos de igual valor. Las mesas negociadoras de los convenios colectivos deberán asegurarse de que los factores y condiciones concurrentes en cada uno de los grupos y niveles profesionales respetan los criterios de adecuación, totalidad y objetividad, y el principio de igual retribución para puestos de igual valor en los términos establecidos en el art. 4 .

Se incorpora una lista abierta de los factores o condiciones considerados relevantes: la penosidad y dificultad, las posturas forzadas, los movimientos repetitivos, la destreza, la minuciosidad, el aislamiento, la responsabilidad tanto económica como relacionada con el bienestar de las personas, la polivalencia o definición extensa de obligaciones, las habilidades sociales, las habilidades de cuidado y atención a las personas, la capacidad de resolución de conflictos o la capacidad de organización, y los criterios a seguir, y sus implicaciones, para llevarla a cabo que son: adecuación, totalidad y objetividad.

Entendiendo por adecuación la acción que implica que los factores relevantes en la valoración deben ser aquellos relacionados con la actividad y que efectivamente concurran en la misma, incluyendo la formación necesaria.

Por totalidad el ejercicio de constatar si concurre igual valor, deben tenerse en cuenta todas las condiciones que singularizan el puesto del trabajo, sin que ninguna se invisibilice o se infravalore.

Y, por objetividad o neutralidad la utilización de mecanismos claros que identifiquen los factores que se han tenido en cuenta en la fijación de una determinada retribución y que no dependan de factores o valoraciones sociales que reflejen estereotipos de género.

A estos efectos, se prevé (disp. final $1^{\mathfrak{a}}$ ) la aprobación de una orden ministerial que apruebe un procedimiento de valoración de los puestos de trabajo, para lo cual se concede

\footnotetext{
${ }^{12}$ Véase: ARAGÓN GÓMEZ, C. y NIETO ROJAS, P.: Planes de igualdad en las empresas. Procedimiento de elaboración e implantación, Wolters Kluwer, Madrid, 2021.
} 
un plazo de seis meses. Estos seis meses se computan desde la entrada en vigor del reglamento, por lo que como ya hemos indicado, vencerán el 14 de octubre de 2021, dos años y siete meses después de que entrara en vigor la exigencia de aplicar estos criterios en la valoración de puestos.

Todos estos cambios legislativos impulsan la puesta en marcha de las dos herramientas, registro y auditorías salariales que junto con la revisión de los sistemas de valoración de puestos de trabajo, deberán ser integradas en todos los Convenios Colectivos.

No obstante, recordemos que en breve se aprobará, bajo el compromiso de la presidenta de la Comisión Europea, Úrsula Von Der Leyen, la Directiva de Transparencia Salarial $^{13}$, con carácter vinculante. Vital para avanzar en la igualdad retributiva entre mujeres y hombres porque prevé sanciones disuasorias en caso de incumplimiento de la norma. Respecto a su contenido de la Directiva el CES ha considerado recientemente la necesidad de que la directiva comprenda en su contenido aspectos significativos. Entre estos destacamos: prohibir las cláusulas de secreto salarial en los contratos, de modo que los trabajadores puedan discutir acerca de los salarios; que se haga pública la evaluación de los puestos de trabajo y así establecer la igualdad salarial por un trabajo de igual valor; obligar a los empresarios a que realicen auditorías para informar sobre los salarios, así como planes de acción anuales sobre igualdad salarial y apoyar a los sindicatos para que negocien con los empresarios la eliminación de la brecha salarial.

Por último, y antes de centrar nuestro estudio en el análisis de la valoración de puestos de trabajo, parece necesario destacar que las diferencias retributivas entre hombres y mujeres son una realidad y que la valoración de puestos de trabajo puede contribuir a reducir por la relación que existe entre ambas fundamentalmente por la infravaloración que implica en el colectivo de mujeres trabajadoras.

Los datos más recientes desvelan que una de cada cinco mujeres con empleo solo va a percibir como máximo el salario mínimo interprofesional. Pero aún es mayor el porcentaje de mujeres en relación a los hombres que solo cobran al mes como máximo 735,90 euros brutos, el 69,57 \% de los 2.291.123 de personas asalariadas que cobran ese salario al mes. Del 1.593.935 de mujeres que cobran solamente el SMI, el 95,18 \% desempeñan una jornada a tiempo completo ${ }^{14}$.

La brecha salarial es evidente, de hecho, la ganancia media anual de las mujeres en 2018, (21.011,89 euros) a 5.726,30 euros de distancia de la ganancia media anual de los hombres en 2018, (26.738, 19 euros), una brecha del 21,41 \%. La última brecha salarial que hemos conocido se sitúa en el 21,41 \%, la más baja de los últimos once años corresponde a los últimos datos disponibles, de 2018, publicada en julio de 2020 por el INE. A pesar de ese descenso las mujeres cobran de media al año, 5.726,30 euros menos que

\footnotetext{
${ }^{13}$ Propuesta de Directiva por la que se refuerza la aplicación del principio de igualdad de retribución entre hombres y mujeres por un mismo trabajo o un trabajo de igual valor a través de

medidas de transparencia retributiva y de mecanismos para su efectivo cumplimiento, Disponible en: https://eurlex.europa.eu/legal-content/ES/TXT/PDF/?uri=CELEX:52021PC0093\&qid=1615286798241\&from=ES

${ }^{14}$ Fuente: Informe Discriminación Salarial 2021, UGT, Disponible en: https://www.ugt.es/Publicaciones/analisis_ de_medidas_y_planes_de_igualdad_en_la_
} 
los hombres. Mientras que el salario medio de las mujeres en 2018 alcanzó la cifra de 21.011,89 euros, por el contrario el de los hombres se situó en 26.738,19 euros al año.

El ritmo al que se corrige la brecha salarial ni es constante ni es significativo, podríamos decir que, si el descenso de medio punto en la brecha salarial en 2018, respecto a 2017 se mantuviera constante en el tiempo se necesitarían 43 años para que la brecha salarial entre sexos desapareciera en España, cuestión aún más prorrogada por la crisis que estamos viviendo.

En 2020, consecuencia de la pandemia los salarios de las mujeres se han visto reducidos en un 14,9\% en el primer semestre del año, frente al. 11,3\% que han perdido los hombres. En términos generales, mientras que las mujeres cobran 35 euros más que los hombres en la horquilla que oscila entre 20.605-25.757 euros anuales, reciben casi 31.000 euros menos cuando las retribuciones escalan por encima de los 103.000 euros al año ${ }^{15}$.

Algunos informes destacan la importancia y repercusión de esta brecha tanto por el coste social y económico directo como por la importancia respecto a la productividad, de hecho, si el salario de la mujer se igualase a la del hombre, la producción aumentaría y se crearían 2,3 millones de trabajo cuestión destacada en el informe de ClosinGap ${ }^{16}$.

Esta situación provoca que las mujeres, a pesar de representar el 51,4\% de la población en edad de trabajar, solamente contribuyan a un 41,5\% del PIB. Esto se entiende principalmente porque las mujeres cobran al año un $21,9 \%$ menos con una diferencia negativa de $5.784 €$. Solo un $8 \%$ de los cargos de presidencia y un $9 \%$ de alta dirección están ocupados por mujeres. Las mujeres trabajan 5,3 M de horas menos al año que los hombres y dedican 2.46 horas más al día al hogar y a la familia.

Puede observarse en esta tabla las diferencias retributivas entre hombres y mujeres por sectores de actividad, en algunos sectores la diferencia es significativa ${ }^{17}$ :

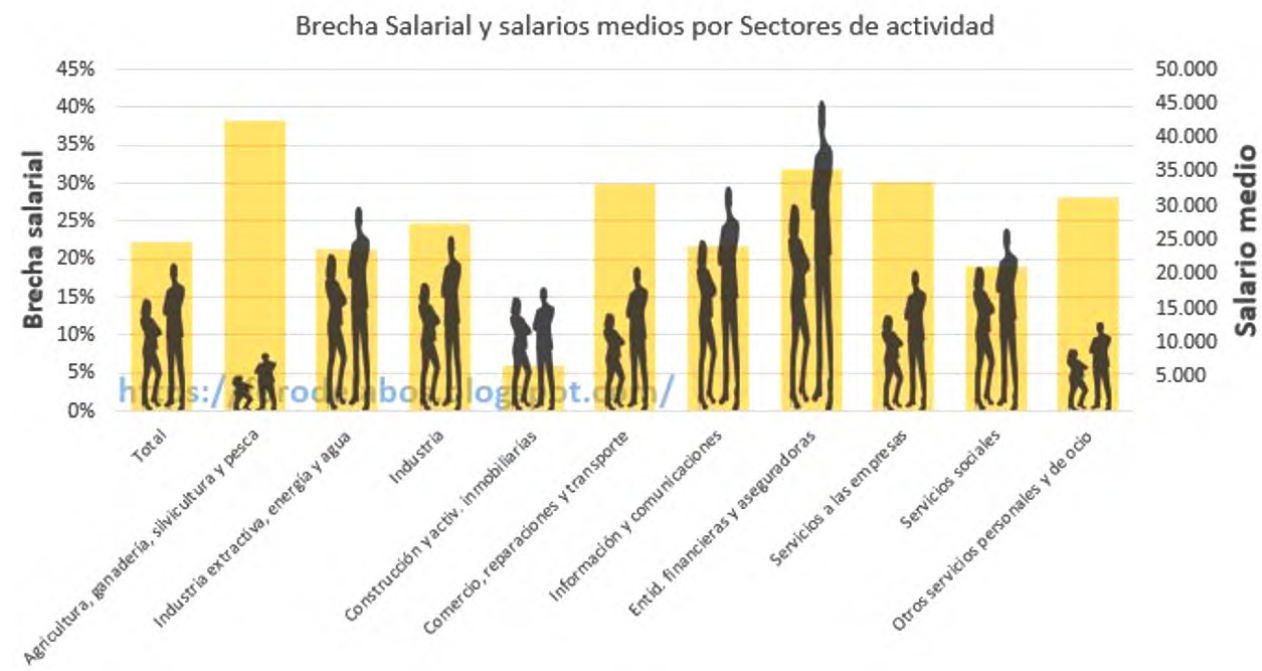

Fuente: Ministerio de Trabajo.

\footnotetext{
${ }^{15}$ CAMACHO M.: Todo lo que quieres saber sobre la brecha salarial de género en España en 2021, Disponible en: https://factorialhr.es/blog/brecha-salarial-genero/\#7.

${ }^{16}$ Fuente: https://closingap.com/actividad/indice-cg/

${ }^{17}$ GIMENO DIAZ DE ATAURI, P.: La valoración de puestos en el reglamento de igualdad retributiva: una nueva huida hacia delante, Disponible en: https://forodelabos.blogspot.com/2020/10/la-valoracion-de-puestos-en-el.html
} 
Ponemos, por tanto, en valor la utilidad como herramienta de la valoración de puestos de trabajo ya que permite realizar un análisis comparativo de los salarios de hombres y mujeres en una organización y, en el caso de que existan diferencias, ayudar a identificar su origen. Para dicho propósito se acompaña de un documento de "Recomendaciones para actuar frente a la brecha salarial de género en una organización"18, detallando el Sistema de Valoración de Puestos de Trabajo (en adelante SVPT) que proporcione un valor a cada puesto, y permita asignarle una retribución acorde a dicho valor.

En concreto, como herramienta de autodiagnóstico de brecha salarial de género: "Sistema de valoración de puestos de trabajo en las empresas con perspectiva de género" que ofrece a las empresas la posibilidad de diseñar su propio SVPT incorporando la perspectiva de género, y utilizarlo para valorar sus puestos de trabajo, determinar un sistema de retribución y compararlo con la realidad de su organización.

\section{Valoración de puestos de trabajo: concepto y clasificación}

\subsection{Concepto}

Entendemos por valoración del puesto de trabajo aquella actividad consistente en determinar el valor de un puesto dentro de una organización empresarial, en relación con los demás puestos de la misma. Es decir, es una forma de ponderar la importancia relativa de un puesto dentro de la organización empresarial y, así, determinar su remuneración económica.

Se desarrolla mediante el análisis y ponderación cuyo objetivo es determinar con precisión el valor relativo de las diferentes funciones de un puesto de trabajo, con el objetivo de establecer una clasificación justa del conjunto de funciones desempeñadas que, además, pueda tomarse como base para la fijación de salarios adecuados.

La determinación del salario y a la adecuación al puesto de trabajo es una tarea imprescindible que debe desarrollarse respetando el principio de igualdad, en este sentido, los salarios no deben ser fijados aleatoriamente.

Mediante la valoración de los puestos de trabajo se puede dimensionar el alcance y las implicaciones de un puesto de trabajo en concreto, ponderarlo en relación a otros puestos de la empresa y, a partir de ahí, asignar un valor, facilitando así detectar cualquier tipo de discriminación indirecta que pudiera darse.

Este procedimiento permite determinar el valor de los puestos de trabajo en una empresa y ponerlos en relación con los requerimientos personales necesarios para su desempeño, como el alcance y las condiciones en las que se desarrollan, con independencia de las personas que ocupen el puesto de trabajo. Se sitúa cada puesto en una escala ordenada que obedece a un número limitado de valores determinados, niveles y categorías que se agrupan

\footnotetext{
${ }^{18}$ Disponible en: http://www.igualdadenlaempresa.es/HerramientaBrecha/Recomendaciones_frente_a_Brecha_Salarial_Genero_16.2.15.pdf
} 
en los denominados grupos profesionales. No olvidemos que la evaluación por desempeño se relaciona normalmente con la configuración del salario variable, y éste, como instrumento, en ocasiones, para la retención del talento. Si se aplica únicamente para algunos puestos, en la mayoría de los casos, puestos masculinizados, puede producir discriminación de género. Por ello, la aplicación a todos los puestos de la organización implica una igual valoración por el desempeño de todos sus profesionales independientemente de su género.

Evidentemente, la valoración sirve y tiene como fin asignar los niveles retributivos agrupando puestos de valor similar y estableciendo intervalos que limitan los grupos o niveles salariales que se detallan posteriormente en los convenios colectivos puesto que es objeto de su regulación

La definición de la valoración de puestos de trabajo debe ser adecuada y basada en criterios realistas y generales aplicables a todos los puestos para que la valoración sirva para corregir o evitar discriminaciones.

Por esto, es necesario que los procedimientos de valoración sean neutros, esto significa establecer parámetros objetivos de valoración absoluta. Estos parámetros son los que nos permiten afirmar que se trata de una herramienta adecuada y eficaz para el control de la discriminación y la corrección de malas prácticas en la materia.

Para que un sistema de valoración de puestos de trabajo sea neutro y objetivo, por ello, garantice la igualdad se debe realizar cumpliendo unos parámetros básicos que a continuación haremos referencia.

Es esencial que antes de la valoración se establezcan las fases del proceso que sustancialmente son las siguientes: determinación de los puestos de trabajo que componen la organización, es decir, la estructura empresarial, descripción de los mismos, evaluación y clasificación, así como los objetivos.

Por un lado, nos referimos a los aspectos de planificación del procedimiento. En concreto, la valoración parte de un listado de puestos y jerarquización que queda sujeta a cada empresa u organización. Tienen que establecerse los puestos de referencia. Definir de forma clara los factores de tal forma que se escalen y puntúen dichos factores. Una parte esencial es el establecimiento de la forma de evaluación de los factores en función de las cualidades de los trabajadores.

Por otro lado, es necesario determinar un lenguaje adecuado, así como garantizar que dos trabajadores distintos deben obtener el mismo resultado o equivalente.

Finalmente, en la conclusión del procedimiento de valoración se significan los resultados de forma transparente indicando puesto y puntos obtenidos.

El desarrollo del proceso bajo el cumplimiento de estos parámetros garantiza la neutralidad y objetividad, con este fin, se consigue no discriminar con relación a los géneros. A los efectos de la eficacia de la valoración debemos tener en cuenta que si este proceso no es neutro, puede desarrollarse una clara discriminación indirecta, ya que los descriptores de valoración son fundamental y tradicionalmente masculinos. La garantía de la igualdad se materializa mediante la ejecución de dos ejes fundamentales.

En primer lugar, en cuanto al proceso se debe aplicar la valoración a todos los puestos, deben incluirse puestos en los mayoritariamente están ocupados por mujeres, se de- 
ben utilizar factores referidos a características tanto femeninas como masculinas y no se debe favorecer con ellas a ningún grupo.

En segundo lugar, en lo referido a los recursos humanos, deben participar evaluadores de los dos sexos con conocimientos específicos en igualdad y desarrollo de valoraciones.

Es una realidad que se han venido utilizando elementos tradicionalmente masculinos de carácter no neutro para valorar los puestos de trabajo, factores como fuerza, esfuerzo físico, etc. $\mathrm{O}$ bien aspectos relacionados con sociedades machistas como la disponibilidad horaria, entendiendo que es un factor generalizado en los hombres y, por el contrario, no tanto en las mujeres debido a la asunción de cargas familiares.

De la misma forma que en aspectos tan significativos como: la estructura salarial y la cuantía retributiva. Esta cuestión ha sido objeto de análisis por la doctrina científica identificando la determinación de esas cláusulas en los convenios colectivos ${ }^{19}$ y poniendo de relieve la necesidad de corrección. De hecho, algunos autores, atribuyen a este elemento el fracaso de la igualdad, en concreto: "a la definición de criterios de valoración del trabajo o, incluso, la integración de los diferenciales de sexo y género -técnicamente conocido- en los reglamentos técnicos de prevención de riesgos laborales" ${ }^{20}$.

Todo ello, desde la perspectiva de los beneficios que aporta al sistema de relaciones laborales. Fundamentalmente porque facilita la valoración de riesgos laborales ajustada a la realidad de cada puesto, favorece y mejora la gestión de recursos humanos y el aprovechamiento del talento, permite el diseño de una clasificación profesional más flexible y adaptada a la realidad y las necesidades de la empresa, frente a un sistema de clasificación profesional rígido e inflexible que impide la adaptación al mercado de trabajo y, como no, visibiliza situaciones de discriminación y permite su corrección.

La valoración de puestos de trabajo se realiza en base al concepto básico de trabajos de igual valor que se fundamenta en un principio básico: el principio de igualdad de retribución que se refiere no sólo a eliminar toda discriminación retributiva por razón de sexo para un mismo trabajo sino para un trabajo al que se atribuye un mismo valor; es decir, deben superarse las desigualdades retributivas no sólo en los trabajos iguales sino también en trabajos diferentes siempre que éstos tengan el mismo valor.

De forma breve señalar, que este principio ya ha sido delimitado por la jurisprudencia comunitaria que ha delimitado las nociones de "igualdad de retribución para el mismo trabajo" o de "igualdad de retribución para un trabajo de igual valor". Para su interpretación es relevante referirse al caso Angestelltenbetriebsrat der Wiener Gebietskrankenkasse donde el TJUE señala, respecto de la expresión "mismo trabajo" que el desempeño de una misma actividad por personas con diferentes cualificaciones profesionales exige constatar si dichos trabajadores efectúan el mismo trabajo, sobre la base de una comparación de criterios objetivos tales como los elementos relativos a la naturaleza de las tareas que pueden encomendarse a cada uno de dichos grupos de trabajadores, los requisitos de

\footnotetext{
${ }^{19}$ PONS PEREA, E,: La legislación y la negociación colectiva como garantías generales de la igualdad retributiva, en el Futuro sin brecha, Bomarzo, 2015, págs. 40 a 46.

${ }^{20}$ GARRIGUES GIMÉNEZ, A.: “Las nuevas relaciones de trabajo: una perspectiva de género”, Revista de Treball, Economia i Societat, núm. 92, Enero 2019.
} 
formación exigidos para su ejercicio y las condiciones laborales en las que dichas tareas se efectúan ${ }^{21}$.

Para poder establecer la categoría de mismo valor de un trabajo la herramienta más precisa es la de la valoración de puestos de trabajo mediante el cual se establece un sistema de clasificación profesional por grupos y puestos de trabajo determinando retribuciones específicas.

Los sistemas de clasificación o de evaluación profesional para determinar salarios, deben basarse en criterios no discriminatorios; esto es, deben tener en cuenta aptitudes comunes a ambos sexos. Como establece el párrafo segundo del art. 1 de la Directiva 75/117/CEE al indicar que "cuando un Estado miembro utilice un sistema de clasificación profesional para la determinación de las retribuciones, estos sistemas deben basarse en criterios iguales para hombres y para mujeres y deben ser diseñados de tal forma que eliminen toda diferencia basada en el sexo". Si bien es cierto que carácter facultativo de los sistemas de clasificación determina que en su ausencia sea el juez nacional el que debe realizar la valoración de los trabajos.

De esta manera, en el caso de la Comisión contra Reino Unido el Tribunal de Justicia estableció que en el Derecho Comunitario no existe la obligación de establecer sistemas de evaluación de los puestos de trabajo, ahora bien, los Estados miembros sí tienen la obligación de asegurar la aplicación del derecho a una remuneración igual por trabajo de igual valor, incluso en ausencia de sistemas de clasificación profesional, siendo el juez nacional competente el que decida sobre la equivalencia de valores entre dos puestos de trabajo. Por ello los Estados miembros tienen que designar la autoridad competente en la materia que pueda establecer y decidir oportunamente si un trabajo tiene el mismo valor que otro ${ }^{22}$ como la competencia otorgada a los órganos jurisdiccionales nacionales ${ }^{23}$.

Recordemos que la propia Comisión Europea ha aportado su definición del principio de igualdad retributiva: "el concepto de igualdad de retribución para un trabajo de igual valor significa que si una mujer desempeña un trabajo que exige el mismo esfuerzo que el de un hombre, aunque el trabajo sea diferente, deberá recibir el mismo salario y las mismas prestaciones a no ser que la diferencia se explique por motivos no discriminatorios". Elementos que, conforme al artículo 28.1 del ET, configuran el principio de igual retribución por trabajos de igual valor y que, como se sabe, vincula a todas las empresas, independientemente del número de personas trabajadoras, y a todos los convenios y acuerdos colectivos. Así, se entenderá que un trabajo tendrá igual valor que otro cuando sean equivalentes.

Esta equivalencia se mide teniendo en cuenta unos elementos determinantes. En primer lugar, la naturaleza de las funciones o tareas efectivamente encomendadas, esto

${ }^{21}$ STJCE de 11 mayo 1999 [TJCE 1999, 101], Angestelltenbetriebsrat der Wiener Gebietskrankenkasse, Asunto 309/97, aps. 18 y 23).

${ }^{22}$ STJCE de 6 julio 1982, Comisión contra Reino Unido, Asunto 61/81, aps. 8 y 13.

${ }^{23}$ SSTJCE de 31 mayo 1995 [TJCE 1995, 79], Royal Copenhagen, Asunto 400/93, ap. 42; SSTJUE 2004\138 27 mayo 2004, Caso Lakeberg Westfalen; 2002 \372, 12 diciembre 2002, Caso Hohorst GmbH Finanzamt Steinfurt; $2000 \backslash 5930$ marzo 200, Caso Jämställdhetsombudsmannen Örebro läns landsting; 1999\101 11 mayo 1999, Caso Angestelltenbetriebsrat der Wiener Gebietskrankenkasse Wiener Gebietskrankenkasse,; 1999\21 9 febrero 199, Caso Regina Secretary of State for Employment; 1998\142 17 junio 1998, Caso Kathleen Hill y otros The Revenue Commissioners y otros, 
es, cuando sea equivalente el contenido esencial de la relación laboral, tanto en atención a lo establecido en la ley o en el convenio colectivo como en atención al contenido efectivo de la actividad desempeñada. En segundo lugar, las condiciones educativas, es decir, las que se correspondan con cualificaciones regladas y guarden relación con el desarrollo de la actividad. En tercer lugar, las condiciones profesionales y de formación, o sea, aquellas que puedan servir para acreditar la cualificación de la persona trabajadora, incluyendo la experiencia o la formación no reglada, siempre que tenga conexión con el desarrollo de la actividad. Y, en cuarto lugar, las condiciones laborales y los factores estrictamente relacionados con el desempeño, entendiéndose por tales aquellos diferentes de los anteriores que sean relevantes en el desempeño de la actividad.

En conclusión, el término igualdad de retribución por razón de sexo significa que a hombres y mujeres les corresponde el mismo salario, siempre que trabajen en los mismos puestos, o incluso en diferentes, cuando tengan el mismo valor, y donde el alcance de la valoración de los puestos de trabajo corresponderá al juez nacional en cada caso concreto. El Tribunal de Justicia ha declarado que para apreciar si unos trabajadores realizan un mismo trabajo o un trabajo al que se puede atribuir un mismo valor debe comprobarse si, habida cuenta de un conjunto de factores ${ }^{24}$. Entre los que ha determinado: la naturaleza del trabajo, las condiciones de formación y las condiciones laborales, si este conjunto es aplicable entonces puede considerarse que dichos trabajadores se encuentran en una situación comparable ${ }^{25}$. Es decir, el alcance de los conceptos de "mismo trabajo", "mismo puesto de trabajo" y "trabajo de igual valor" a los que se refiere la normativa comunitaria revisten un carácter puramente cualitativo, en cuanto están exclusivamente ligados a la naturaleza del trabajo efectivamente realizado por los interesados ${ }^{26}$.

En todo caso, en el marco de una discriminación salarial indirecta, incumbe al empleador aportar una justificación objetiva de la diferencia de retribución apreciada entre los trabajadores que se consideran discriminados y las personas de referencia. La justificación ofrecida por el empleador de la diferencia de retribución que pone de manifiesto una apariencia de discriminación por razón de sexo debe guardar relación con las personas de referencia que, debido a que su situación se caracteriza por datos estadísticos válidos referidos a un número suficiente de individuos, que no constituyen la expresión de fenómenos meramente fortuitos o coyunturales y que, de manera general, resultan significativos, han sido tomadas en consideración por el órgano jurisdiccional nacional para apreciar dicha diferencia.

El interés por mantener unas buenas relaciones laborales puede ser tomado en consideración por el órgano jurisdiccional nacional entre otros elementos para apreciar si las diferencias entre las retribuciones de dos grupos de trabajadores se deben a factores objetivos y ajenos a cualquier discriminación por razón de sexo y son conformes con el principio de proporcionalidad.

\footnotetext{
${ }^{24}$ SSTJCE en este mismo sentido respecto a la igualdad de retribución entre hombres y mujeres:

${ }^{25}$ STJCE $2013 \backslash 67$ Caso Margaret Kenny contra Minister for Justice, Equality and Law Reform. Sentencia de 28 febrero 2013.

${ }^{26}$ STJCE de 26 junio 2001, Brunnhofer, Asunto 381/99, ap. 42.
} 


\subsection{Clasificación de modelos de valoración de puestos de trabajo}

Los procedimientos de valoración de puestos de trabajo son de diversa índole, principalmente cualitativos y cuantitativos.

Por un lado, los cualitativos que valoran los puestos de trabajo de forma global, con el fin de ordenar y jerarquizar los puestos de trabajo. Por otro lado, los cuantitativos o analíticos que evalúan los puestos según distintos criterios, que se denominan factores, previamente seleccionados y claramente definidos.

A su vez, pueden clasificarse en procedimientos de comparación de factores, por una parte, y de asignación de puntos por factor, por otra.

Al ser cuantitativos permiten determinar un valor numérico de cada puesto y, por tanto, implica cuantificar la diferencia de valor entre puestos. Se asignan puntos hasta formar un total teniendo en cuenta: conocimientos, aptitudes comunicativas, de relaciones humanas, esfuerzo mental, responsabilidad sobre materiales, etc., y una escala de valoración para cada factor con un número determinado de niveles o grados.

La OIT ha considerado como más adecuado el sistema de puntos por factor, que pertenece al sistema cualitativo o analítico, es considerado como el más adecuado.

\subsubsection{Sistemas cualitativos o globales}

Son procedimientos que comparan los empleos y los clasifican con arreglo a los requisitos de base, sin realizar un análisis detallado de su contenido. De esta forma es posible saber cuál es la importancia de cada puesto, pero no las diferencias de valor entre puestos.

Estos sistemas son de jerarquización o de clasificación.

Por jerarquización se establece una escala de puestos de trabajo, mediante el cual se averigua si un puesto, entendido como unidad — conjunto indivisible de funciones, responsabilidades y tareas-, es igual, superior o inferior a otro. Este procedimiento ordena los puestos de forma descendente (de nivel inferior al superior) o en sentido descendente.

Por clasificación se ordenan los puestos a valorar respecto a una escala con unos niveles o grados ya establecidos. Dentro de una categoría de puestos dada, existen diferencias en los niveles de responsabilidades, funciones y habilidades ejercidas en cada puesto. Cuando estas diferencias son identificadas, pueden ser expresadas en términos de grados definidos, ordenados a partir de los requisitos en grado mínimo dentro de la categoría que se califica y hacia los grados máximos.

\subsubsection{Sistemas cuantitativos o analíticos}

Partiendo del elemento fundamental que es el factor estos sistemas se dividen en sistema de comparación por factores y sistema de puntos por factor.

El sistema de comparación por factores se desarrolla evaluando el puesto, no como unidad, sino mediante un análisis en detalle que permite descomponerlo en "factores" 
que se jerarquizan en orden de importancia. Los puestos no se juzgan mediante una escala descriptiva, sino que se comparan, uno con otro, factor por factor, para determinar su valor relativo. Es un sistema basado en la comparación.

El segundo tipo, el de puntos por factor es un sistema en el que se combinan el procedimiento analítico, que requiere la descomposición de los puestos en factores, con el cuantitativo, que asigna un valor a cada uno de los citados factores.

\section{Elección del procedimiento de valoración de puestos de trabajo}

Los sistemas cualitativos o globales son procedimientos que tienen a su favor que son sencillos en su uso y aplicación, sin embargo, no proporcionan información sobre las diferencias de valor entre puestos y no permiten detectar la influencia de prejuicios o estereotipos de género.

Los sistemas cuantitativos o analíticos son procedimientos que permiten examinar de manera sistemática todos los requisitos de los puestos de trabajo, permiten evaluarlos y compararlos mediante criterios comunes, precisos y detallados. Identifican la diferencia de valor entre puestos, ya que asignan un valor numérico a cada puesto.

La OIT, como ya hemos indicado, ha considerado como más adecuado el sistema de puntos por factor ${ }^{27}$, que pertenece al sistema cualitativo o analítico, es considerado como el más adecuado. Este sistema se basa en cuatro factores denominados base: calificaciones, esfuerzos, responsabilidades y condiciones de trabajo.

Estos factores son necesarios y suficientes para evaluar todos los puestos y todas las empresas, con independencia del sector económico al que pertenezcan, gracias a que la descomposición de los citados factores en subfactores permite tener en cuenta características más detalladas de cada empleo y entidad. En todo caso, deben tenerse en cuenta dos límites significativos que son: el rigor metodológico y la ausencia de prejuicios sexistas.

Entendiendo por rigor metodológico la incorporación de tres espacios de desarrollo: adaptación a la empresa, ausencia de ambigüedad y ausencia de superposición.

El espacio de adaptación a la empresa se realiza en el proceso mediante la selección de empleos que serán comparados, estableciendo los grupos profesionales, las tareas y los tipos de empleo. Es necesario identificar la estructura de la empresa en función de su idiosincrasia estructural ya que de esta depende la elección de los subfactores determinantes para la valoración. Por ejemplo, en una empresa pequeña se suelen utilizar unos ocho subfactores.

La ausencia de ambigüedad en la descripción, propósito y objetivos de cada subfactor. Esto nos lleva a la afirmación de que no pueden existir elementos discordantes ya que en ese caso los evaluadores se encontrarán con dificultades para interpretarlos. Tal sería el caso, por ejemplo, de un subfactor que abarcara al mismo tiempo la manipulación

\footnotetext{
${ }^{27}$ Promoción de la Igualdad salarial por medio de la evaluación no sexista de los empleos, OIT, Disponible en:https:// www.ilo.org/public/libdoc/ilo/2008/108B09_271_span.pdf
} 
de objetos pesados, las posturas de trabajo poco ergonómicas y la atención visual, tres dimensiones muy diferentes del esfuerzo físico.

El último elemento al que hemos hecho referencia es la ausencia de superposición que implica la necesidad de garantizar que un subfactor se tenga en cuenta dos veces ya que puede desequilibrar la balanza y, por tanto, resultar discriminatorio.

Dependiendo del tamaño de la empresa y la diversidad de puestos variará el número y contenido de subfactores. En términos generales podemos establecer como subfactores; calificaciones, esfuerzos, responsabilidades y condiciones de trabajo. Subfactores que parten de un descriptor en condiciones de igualdad y que no diferencian aspectos en función del sexo, por el contrario, describen aspectos neutros y que podemos identificar en términos generales en la siguiente tabla:

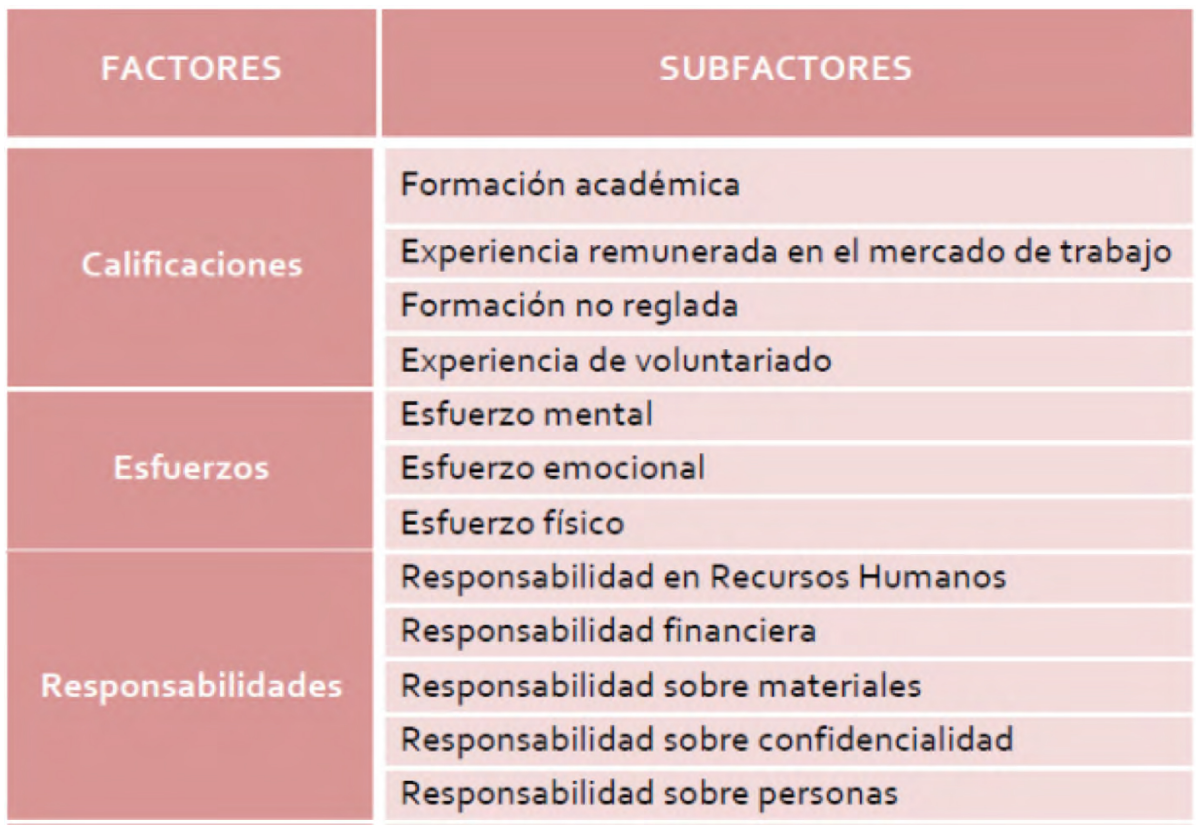

Fuente: Ministerio de Sanidad, Servicios Sociales e Igualdad.

\section{Elementos básicos de la valoración de puestos de trabajo en relación con el gé- nero}

La valoración de puestos de trabajo considera en su conjunto varios elementos: capacidades, esfuerzo, responsabilidad y condiciones de trabajo.

Respecto del primero de los elementos, las capacidades, el análisis se concentra en valores concretos como son: conocimientos, aptitudes e iniciativa.

El segundo de los elementos, el esfuerzo, puede ser físico, mental y emocional. Las responsabilidades se extienden a mando, supervisión de personal, seguridad de materiales, información y seguridad y bienestar de los trabajadores.

Por último, las condiciones de trabajo. Conforme a estos elementos la valoración se realiza puesto por puesto analizando las determinaciones de cada uno y de cada trabajador. 
Como ya hemos hecho referencia este proceso tiene como eje fundamental determinar el valor relativo de los puestos de trabajo de una organización y en nuestro análisis pretendemos establecer su relación con la igualdad de valor entre dos puestos independientemente del sexo de los trabajadores con el objeto de combatir la desigualdad garantizando que la evaluación se lleva a cabo sin sesgos de género.

En este sentido, resulta crucial que se valore el puesto en función de las tareas y no de la persona que lleva a cabo las funciones, en otras palabras, de forma neutra estableciendo las características masculinas y femeninas sin favorecer unas sobre otras. Incluyendo en el proceso expertos en igualdad y valoración de puestos de trabajo y apartados de estereotipos de género. Para que esto sea efectivo y práctico es necesario crear estructuras de puestos de trabajo que no caigan en límite establecido derivado de una medida común entre los trabajos desempeñados por mujeres y los desempeñados por hombres. Esa medida común alejada de los medidores clásicos que diferencian entre trabajos feminizados y trabajos masculinizados. El objetivo es valorar el puesto de trabajo independientemente de la persona que lo ocupa y más aún independientemente del sexo del trabajador que lo desempeña.

De la misma forma debe observarse un cuidado expreso en la denominación de los puestos que se debe realizar conforme a criterios objetivos no sujetos a la feminización o masculinización.

Los descriptores del puesto de trabajo se tienen que ajustar a la determinación de capacidades y responsabilidades concretas para el puesto que se alejen de estereotipos vinculados a la condición de hombre o la condición de mujer. El proceso en la fase de comprobación se detiene en establecer en cada puesto las capacidades de: atención, organizativas, de responsabilidad, de coordinación o de calidad entre otras. Así como los factores de capacidad referidos a educación, formación y experiencia profesional. Lo mismo que otros elementos calificados como "ligero o pesado"28.

\section{Elementos relevantes de la valoración de puestos de trabajo relacionados con el género}

Llegados a este punto es imprescindible establecer las bases fundamentales en la valoración de puestos de trabajo con el fin de impedir la discriminación por género.

En este sentido estableceremos aquellos aspectos conforme a los cuales la valoración de puestos de trabajo es una herramienta eficaz para garantizar la igualdad de género desde el control en los convenios colectivos y los planes de igualdad ${ }^{29}$.

Es importante destacar, en términos generales, los beneficios que desde la perspectiva de género aporta la valoración de puestos de trabajo que nos parece importante señalar.

${ }^{28}$ COVES, A.M.; COROMINAS, A.; LUSA, A. y MARTÍNEZ, C.: La valoración de puestos de trabajo y la discriminación retributiva, Disponible en: https://recerca.upc.edu/giopact/publicacions/Documents/comunicacin_crdoba.doc/at_ download/file y La discriminación salarial de la mujer y su relación con la valoración de puestos de trabajo", Disponible en: https://recerca.upc.edu/giopact/ca/projects/docs/n_dit3.pdf

${ }^{29}$ Análisis de medidas y planes de igualdad en la negociación colectiva, UGT, 2010, Disponible en: https://www.ugt. es/Publicaciones/analisis_de_medidas_y_planes_de_igualdad_en_la_ 
En primer lugar, visibiliza la concentración de sexos en determinadas profesiones, tareas o categorías profesionales.

En segundo lugar, permite una adecuada, y ajustada a la realidad, valoración de tareas, funciones y puestos desempeñados tradicionalmente por mujeres. Que, en ocasiones, como consecuencia de ser entendidos como una prolongación del trabajo doméstico y reproductivo, han sido infravalorados y menospreciados en el mercado de trabajo.

Y, en tercer lugar, contribuye y posibilita la corrección de la brecha salarial de género, al ponderar los puestos de trabajo de acuerdo con su valor y las funciones que se desempeñan y no según el sexo de la persona que los desempeña.

El impacto de la valoración de puestos de trabajo en garantizar la igualdad de género y, por ello, evitar la discriminación indirecta se puede hacer referencia a cuestiones que en los convenios colectivos y en los planes de igualdad se deben vigilar con especial cuidado con el fin de evitar desigualdades entre mujeres y hombres.

\subsection{La equidad en niveles profesionales y en puestos de trabajo}

La determinación de los grupos profesionales y los puestos de trabajo tiene que realizarse sin que se produzca una asociación implícita entre puesto y sexo; esto supone una estructura independiente del sexo de la persona que lo ocupe. Esto puede significar agruparlos o dividirlos, dependiendo de la estructura empresarial.

Pongamos como ejemplo varios supuestos referidos a este aspecto.

Es imprescindible la asignación de niveles de modo equitativo, fijándose de modo exclusivo en la descripción del puesto que ha de ser completa, sin considerar si dichos puestos están masculinizados o feminizados. Cuidando que no se apliquen sesgos de género asignando a los puestos feminizados niveles de exigencia inferiores y por ello valoraciones inferiores.

Es importante agrupar los puestos de valores similares de forma que se utilicen requerimientos equivalentes de capacidad, esfuerzo, responsabilidad y condiciones laborales con unas mismas bandas salariales. Esto facilita la neutralización de los sesgos de género en la retribución, al equiparar salarialmente puestos masculinizados y feminizados.

Podemos considerar algunos ejemplos a continuación.

En primer lugar, colectivo de mujeres y hombres, cuyas funciones y responsabilidades son comunes o polivalentes. Se determinan dos puestos diferentes, uno con mayoría de mujeres y otro con mayoría de hombres. Si se detectan diferencias salariales o se asignan bandas salariales distintas puede establecerse como causa que se trata de puestos diferentes cuando en realidad es el mismo puesto.

En segundo lugar, si dos grupos de mujeres realizan funciones diferentes, pero que se agrupan en un mismo puesto; el análisis de sus retribuciones no se ajustará a la realidad puesto que es necesario establecer una retribución en función del puesto de trabajo correspondiente a las funciones desempeñadas. 


\subsection{Universalidad de factores y equilibrio de ponderación}

Los factores seleccionados para la valoración de puestos de trabajo, a los que ya hemos hecho referencia tienen que ser de carácter universal. Esto garantiza que pueden ser aplicables para su ponderación y nivelación a todos s los puestos de trabajo de la organización.

El uso de ponderaciones diferentes para cada puesto haría que el valor de cada uno se midiera con una escala diferente, imposibilitando el concepto de igual salario para igual valor y descartando el principio de proporcionalidad en la asignación de cuantías. Evidentemente la baremación en función del valor comporta asignar los pesos en función del valor objetivo aportado por cada factor a la organización. Sería, por supuesto, discriminatorio asignar un peso muy alto al esfuerzo físico, típicamente más presente en puestos masculinizados, y uno muy bajo a las habilidades de comunicación, típico de puestos feminizados.

Sobre esta cuestión el TJUE consideró que un sistema valoración de puestos de trabajo donde uno de los criterios a tener en cuenta sea el del esfuerzo o el de la carga muscular no es necesariamente discriminatorio, por el solo hecho de que implique cualidades que poseen de forma más habitual los hombres. La valoración de puestos de trabajo sobre la base de la fuerza física no constituye una discriminación si objetivamente se mide la fuerza porque el trabajo así lo exige y no intervienen otras consideraciones. Por el contrario, sí sería discriminatorio un sistema de clasificación basado en la fuerza cuando ésta no sea necesaria para ejecutar el trabajo valorado ${ }^{30}$.

Lo mismo respecto a la ponderación de los factores; considerar más importantes unos factores que otros es un claro supuesto de discriminación que, por supuesto, repercute en la discriminación efectiva hacía la mujer. En la mayoría de las ocasiones se tienen en cuenta factores que favorecen el reconocimiento de actividades masculinizadas.

\subsection{Establecer denominaciones neutras para los puestos de trabajo}

Las denominaciones siempre que sea posible, tiene que realizarse de forma que los términos utilizados sean neutros o de carácter colectivo, en el supuesto de que esta premisa no pueda aplicarse deben emplearse los dos géneros gramaticales.

El objeto es evitar consideraciones previas que inducen a la discriminación de la mujer, como asociar un sexo a un puesto, el caso más claro lo encontramos en las cuidadoras, limpiadoras o en el servicio del hogar familiar, es decir, estereotipos de género, que provocan infravaloración de los puestos de trabajo que ocupan las mujeres.

Bastante común en profesiones feminizadas como las relacionadas con las labores domésticas, siendo el 97,7\% del porcentaje total, o bien la enseñanza, con una presencia del $96,7 \%$ de mujeres. Somos capaces de reconocer puestos de trabajo con carácter dis-

${ }^{30}$ STJCE de 1 julio 1986, Rummler [TJCE 1986, 107], Asunto 237/85, ap. 17. 
criminatorio como por ejemplo: "la enfermera" frente "al médico" "la cuidadora" o muy típico "la limpiadora".

\subsection{Puestos de trabajo feminizados de la organización}

Con el objeto de establecer una valoración de puestos equilibrada y garantizadora de la igualdad de género basada en la objetividad y neutralidad, es muy importante cuidar la selección de puestos feminizados considerados de referencia en la estructura que sean de distinto nivel en los que predomine la ocupación de mujeres.

La elección de estos puestos de referencia en la valoración de puestos sirve para establecer modelos que sirven para determinar el resto de puestos valorados. Al considerar desde un inicio puestos feminizados, se asegura que las características asociadas a los mismos se refieran a factores determinantes, mientras que de otro modo los puestos en los que están mayoritariamente las mujeres serían valorados posteriormente y las características a ellos asociadas tendrían un valor secundario con sesgos de género evidente.

\subsection{Exhaustividad en la descripción de los puestos de trabajo}

Asociado al elemento anterior, es necesario ser rigurosos en las características que se asocian a cada puesto de trabajo, asegurando que el detalle de la descripción y los factores aplicados sean los mismos.

Si la descripción no responde a estos parámetros en el caso de puestos feminizados existe el riesgo de "obviar" las características o tareas asociadas a los estereotipos de género para las mujeres, lo que provoca en consecuencia que la valoración se haga de forma posterior, probablemente, desde una perspectiva subjetiva y, por tanto, no igual despreciando el concepto de igual valor.

El conjunto de factores tiene que ser completo, es decir, debe cubrir todas las características relevantes del puesto. Los factores deben ser independientes, no redundantes, puesto que si una característica es tenida en cuenta por más de un factor se le está otorgando implícitamente mayor importancia produciendo un claro desequilibrio.

Un ejemplo típico se produce en empresas pequeñas, pongamos como ejemplo, una empresa que tiene tres puestos de recepción de llamadas, ocupados por mujeres. Se entiende que, entre las capacidades necesarias para el puesto de recepción de llamadas, se encuentran habilidades de comunicación y empatía. Se trata de factores denominados de habilidades asociados a la descripción del puesto, pero esta valoración debe realizarse objetivamente y de forma neutra.

En suma, considerar que es un factor asociado al puesto de trabajo, pero no a la condición de género, de lo contrario se produciría una infravaloración de las habilidades y del puesto de trabajo. 


\subsection{Repetición de descripciones y necesidades en los puestos de trabajo}

La valoración doble o las repeticiones en la descripción suponen una doble valoración que se puede evitar homogeneizando las denominaciones de las características de los puestos. Si en la descripción de puestos aparecen tareas o funciones que son en realidad la misma, o requieren de una misma habilidad o conocimiento, esto puede hacer que sea valorada dos veces en lugar de una.

Este hecho puede suceder en los puestos masculinizados, ya que los hombres, por estereotipo tradicional social, consideran que su trabajo es merecedor de valoración, en mayor medida que las mujeres. Si bien es cierto que no es una premisa generalizada se ha identificado en algunas ocasiones.

Téngase en cuenta que, si aparecen tareas o funciones equivalentes con diferentes nombres, puede que den lugar a una connotación y valoración distinta. Por ejemplo, denominar "dirección" a la gestión de equipos o departamentos cuando estos puestos están ocupados por hombres, y referirse a la misma como "coordinación" cuando se trata de puestos ocupados por mujeres. En este caso se produce de nuevo una infravaloración.

En consecuencia, la aplicación sistemática de todos los factores a todos los puestos perjudica claramente la objetividad ya que puede suceder que en puestos feminizados determinados factores no se consideren relevantes, debido a un sesgo de género. Por ejemplo, en el caso de las condiciones de trabajo, las personas que ocupan puestos de atención al cliente pueden tener unas condiciones de trabajo que a priori pudieran considerarse buenas (trabajo en oficina, no desplazamientos), pero que en cambio pueden producir ciertos perjuicios que es preciso considerar que suponen riesgos psicosociales: reducidas dimensiones del puesto físico de trabajo, limitaciones en las pausas, ruido de ambiente, etc.

La objetividad y neutralidad quedan garantizadas en mayor medida cuando los resultados se miden cuantitativamente, es decir, cuando se evalúa por pares y con personal cualificado experto en género. De forma clara la objetividad evita la arbitrariedad que puede estar influida por los estereotipos de género.

Al definir los factores también es importante cuidar que no se mida el mismo aspecto bajo dos factores supuestamente distintos; por ejemplo, autonomía en la toma de decisiones e iniciativa en la resolución de problemas.

\subsection{Equidad de género respecto de las circunstancias o condiciones del puesto de trabajo}

Otro aspecto significativo es la necesidad de equidad de género en la asociación de circunstancias que generan complementos salariales, tanto de modo automático, como la peligrosidad o la penosidad, como en circunstancias concretas, como puede ser el complemento de turnicidad, o bien el de disponibilidad.

En este caso la propia determinación de qué es objeto cada complemento puede incluir un sesgo de género. Por ejemplo, tradicionalmente en la industria se considera remunerable peligrosidad en el manejo de maquinaria que con carácter permanente se 
considera asociado a puestos masculinizados mientras que el manejo de productos tóxicos y peligros de limpieza se asocia normalmente a puestos feminizados. Curiosamente los trabajos con sustancias tóxicas y peligrosas son tradicionalmente masculinos cuando no son productos de limpieza.

De la misma forma, si la asociación de circunstancias se produce de forma inversa, es decir, si se asocian circunstancias que no tienen relación con el puesto de trabajo estamos ante un claro sesgo de género. Por ejemplo, cuando se asocia a un puesto la necesidad de flexibilidad horaria y se parte de la consideración que los hombres tienen de partida mayor disponibilidad horaria, por ejemplo, para prolongar la jornada. En ese caso si dicha consideración no es precisa en el puesto de trabajo y no existe relación directa con las tareas y el desempeño del mismo, se considera un factor de discriminación hacía la mujer. Esta circunstancia se resume en el premio al presentismo frente a la productividad que es un factor neutro y equilibrado.

\subsection{Garantía de retribución por circunstancias asociadas al puesto de trabajo}

Es preciso también considerar, para evitar discriminaciones indirectas, el hecho de considerar remunerables ciertas circunstancias asociadas al puesto, pese a ser perfectamente justificables en el puesto de trabajo que se describe. En concreto, se precisa adecuar las condiciones, o modificar su definición, para procurar que sean y atribuibles por igual y de forma indiferente a mujeres y hombres.

Tomemos como ejemplo el caso de las horas extras donde la brecha alcanza el 78\%, la disponibilidad, los desplazamientos o la formación, si se realiza fuera del horario de trabajo, las mujeres pueden tener, en principio, una posición de desventaja, dado que puede resultarles más complejo realizarlas por las denominadas cargas familiares atribuibles en su mayoría a la mujer como un rol tradicional en nuestra sociedad.

Por ejemplo en el caso de la maternidad el TJUE en $1999^{31}$ estableció que el principio de igualdad de retribución consagrado en el art. 119 del Tratado CE y los arts. 117 a 120 del Tratado CE, sustituidos por los artículos 136 CE a 143 CE, no se opone al pago de una asignación a tanto alzado reservada únicamente a las trabajadoras que inician su permiso de maternidad, siempre que dicha asignación esté destinada a compensar las desventajas profesionales que la interrupción del trabajo supone para ellas.

0 en el supuesto específico de retribuciones asociadas a primas especiales anuales en determinados puestos de trabajo, el TJUE considera que debe interpretarse en el sentido de que constituye una discriminación indirecta por razón de sexo el hecho de que las personas que ejercen actividades por cuenta ajena con un horario normal inferior a quince horas semanales y una retribución normal que no supera una determinada fracción de la base mensual de referencia y que, por ello, están exentas del seguro social obligatorio,

\footnotetext{
${ }^{31}$ STJCE de 16 de septiembre 1999, 1999\199. Caso Oumar Dabo Abdoulaye y otros contra Régie nationale des usines Renault SA.
} 
queden excluidas por un convenio colectivo de la concesión de una prima especial anual, prevista por éste, que se aplica con independencia del sexo del trabajador, pero que afecta de hecho a un porcentaje considerablemente mayor de mujeres que de hombres ${ }^{32}$.

Lo mismo sucede con el plus de asistencia, puede ser una fuente de discriminación por razón de género, según que se entienda por ausencia. De ahí la importancia de la negociación colectiva en la determinación del concepto de ausencia.

${ }^{32}$ STJCE $1999 \backslash 1799$ de septiembre 1999. Caso Andrea Krüger contra Kreiskrankenhaus Ebersberg. 